\title{
On Computing Task-Oriented Grasps
}

\author{
Sahar El-Khoury, Ravin de Souza, Aude Billard \\ Learning Algorithms and Systems Laboratory (LASA), \\ Ecole Polytechnique Federale de Lausanne (EPFL), Switzerland
}

\begin{abstract}
This paper addresses the problem of optimal grasping of an object with a multi-fingered robotic hand for accomplishing a given task. The task is first demonstrated by a human operator and its force/torque requirements are captured through the usage of a sensorized tool. The grasp quality is computed through a task compatibility criterion. Grasp synthesis is then formulated as a single constrained optimization problem, generating grasps that are feasible for the hand's kinematics by maximizing the corresponding task-oriented quality criterion and ensuring grasp stability. The method was validated on a human hand model and is shown to be easily adapted to different hand kinematic models.
\end{abstract}

Keywords: Grasping, force-closure, hand kinematics, Task modelling

\section{Related work}

One fundamental challenge in robotic grasping is: given a particular hand, an object and a task to perform, what is the optimal grasp of the object permitting to efficiently accomplish the task? In order to answer this question, two main issues need to be addressed: 1) capturing and modelling the task requirements and 2) finding the best hand configuration that is adapted to the task.

\subsection{Task modelling}

Few approaches in the literature addressed the problem of task modelling. The wrenches that are expected to occur for a given task are referred to as the Task Wrench Space (TWS). Li and Sastry [12] showed that the choice of a grasp should be based on its capability to generate wrenches that are relevant to the task. Assuming the task and the object geometry to be known, they computed the object trajectory while performing the task before the grasping action occurred and based on a human experience, they modeled the task by a six-dimensional ellipsoid, called task ellipsoid, in the object wrench space. These ellipsoid's principal axes were determined intuitively by forces/torques requirements directions. Considering the difficulty of pre-computing the object trajectory to accomplish the task, the authors in [18] assumed the task to be unknown. In this case, the probability for every wrench direction to occur as a disturbance is equal and the TWS

Email addresses: sahar.elkhoury@epfl.ch (Sahar El-Khoury), ravin.desouza@epfl.ch (Ravin de Souza), aude.billardeepfl.ch (Aude Billard)

Preprint submitted to Robotics and Autonomous Systems

November 7, 2014 
is modeled as a unit sphere. However, modeling the TWS with a unit sphere has no physical interpretation; in order to accomplish a task, the forces and their corresponding torques act on the object surface and consequently the TWS varies with the object shape. Thus, the TWS is not uniform and Pollard [16] introduced the notion of Object Wrench Space (OWS) incorporating the object geometry in the computation of the TWS. The OWS is a general model over any task and takes into account all possible disturbances on the object surface. Borst et al. [4] approximated the OWS with an ellipsoid in order to analytically model the TWS. This enabled the authors to obtain a physically motivated, task independent description of the TWS. More recently and in order to consider a specific task constraints, the authors in [5] introduced the Functional Wrench Space (FWS) which is defined as the set of wrenches exerted on an object through a sequence of taskoriented demonstrated grasps in a virtual reality environment. The FWS is a subset of the global OWS, is task-dependent as it is defined based on a set of exemplar grasps appropriate for the task but does not take into account the wrenches exerted on the object all along the task.

\subsection{Task-oriented quality criteria and grasp synthesis}

Based on these models, researchers proposed several criteria to measure the quality of a taskoriented grasp. The convex hull over the set of all wrenches that the manipulator can exert on the object for a given grasp is defined as the Grasp Wrench Space (GWS). Li and Sastry [12] defined their quality criterion as the scaling factor that can embed the largest task ellipsoid to the grasp wrench space. Borst et al. [4] defined this criterion as the largest scaling factor for a given OWS to fit it into a GWS. Aleotti et al. [5] evaluated a grasp by computing a quality metric, defined as the largest factor by which the GWS can be scaled to fit in the demonstrated functional wrench space (FWS). While the authors in $[4,16]$ used these criteria for selecting the optimal grasp among a set of pre-computed grasps, the authors in [12] used the grasp quality criterion to solve the grasp synthesis problem. They formulate grasp synthesis as an optimization problem in order to determine the optimal contact locations on the object's surface without however taking into account the feasibility of their solution for the the hand kinematic constraints. In [5], the authors use grasp quality criterion in order to locally optimize a grasp; given a candidate grasp suggested by a grasp planning algorithm, they find a sub-optimal grasp close to the specified one.

\subsection{Our approach}

The previous approaches present two major limitations. Task modelling has been performed either in a general, task-independent manner $[16,18,4]$ or when trying to take into account the specificity of one particular task, the model was constructed intuitively [12] or based on a virtual environment grasp experience [5]. The second limitation concerns the task-oriented grasp synthesis which was reduced to optimizing either the contact points locations on the object or when taking into account the hand model, to only locally optimizing pre-generated grasps and consequently generating sub-optimal grasps.

In our previous work [11], we proposed a method for generating a variety of grasps by formulating grasp synthesis as a single constrained optimization problem. The computed grasps were feasible for the hands kinematics and were obtained by minimizing the norm of the joint torque vector of the hand ensuring grasp stability. We extend this previous work in three ways: 1) We use a sensorized tool and model the task constraints in terms of force/torque exerted on the object 
all along the task from human demonstration; 2) We conduct an experiment showing that the task model does not depend on the choice of the grasp; 3) We include the task constraints extracted from human demonstration in the formulation of grasp synthesis, modify our objective function to a task-oriented quality measure and generate task compatible grasps. The algorithm was tested on a biologically plausible representation of the human hand.

In the following paragraphs, we derive the steps by which we can include the task-oriented quality criterion, the kinematic constraints of the hand to describe finger placement on the object surface yielding an optimal grasp. Section 2 describes the sensorized tool and its usage in modelling the task wrench space. Section 3 describes the Human hand kinematics and force capabilities model; Section 4 describes briefly the representation of the object surface; Section 5 describes the constraints that must be satisfied to yield stable and high quality task-oriented grasps on 3D objects; Section 6 shows that grasp computation can be formulated as a non-linear optimization problem; Section 7 illustrates the performance of the approach; Section 8 concludes.

\subsection{Notation}

In the following of the document, bold letters represent vectors, capital letters represent matrices and normal letters represent scalars.

$R_{O}=\left(\mathbf{o}_{1}, \mathbf{o}_{2}, \mathbf{o}_{3}\right)=$ orthonormal reference frame attached to the object.

$R_{h}=\left(\mathbf{h}_{\mathbf{1}}, \mathbf{h}_{\mathbf{2}}, \mathbf{h}_{\mathbf{3}}\right)=$ orthonormal reference frame attached to the palm expressed in $R_{o}$.

$\mathbf{d}_{\mathbf{h}}=$ palm's position expressed in $R_{O}$.

$R_{i}^{j}=\left(\mathbf{e}_{\mathbf{i}}^{\mathbf{j}}, v_{\mathbf{i}}^{\mathbf{j}}, \mathbf{r}_{\mathbf{i}}^{\mathbf{j}}\right)=$ orthonormal reference frame attached to joint $\mathrm{i}$ of finger $\mathrm{j}$ expressed in $R_{h}$.

$\mathbf{d}_{\mathbf{k}}^{\mathbf{j}}=$ vector connecting origins of $R_{k}^{j}$ and $R_{k+1}^{j}$, related to the $k^{\text {th }}$ and $(k+1)^{\text {th }}$ links of finger $j$.

$q^{j}=$ number of joints of the $j^{\text {th }}$ finger.

$q=$ number of joints torques for all fingers.

$b=$ number of fingers, also number of contact points.

$\tau_{i \text { max }}^{j}=$ maximal $i^{\text {th }}$ joint torque of the $j^{\text {th }}$ finger.

$\tau=\left\{\tau_{i}^{j}\right\} \in \mathbb{R}^{q b}=$ all hand joint torques.

$J^{j}=$ jacobian matrix of the $j^{\text {th }}$ finger.

$J=\operatorname{diag}\left(J^{1}, J^{2}, . ., J^{b}\right)$.

$G \in \mathbb{R}^{6 \times 3 b}=$ the grasp matrix.

$\mathbf{p}^{\mathbf{j}}=$ contact point located on the $j^{\text {th }}$ finger.

$\mathbf{n}_{\mathbf{p}} \mathbf{j}=$ contact normal at point $\mathbf{p}^{\mathbf{j}}$.

$\mathbf{f}^{\mathbf{j}}=$ force applied by the hand at contact point $\mathbf{p}^{\mathbf{j}}$.

$\mathbf{f} \in \mathbb{R}^{3 b}=$ all the applied forces at all the contact points.

$g(p)=$ implicit surface representing the object shape.

$\mathbf{l}_{\mathbf{i}}^{\mathbf{j}}=i^{t h}$ edge vector of the friction cone centered about the contact point internal normal $\mathbf{n}_{\mathbf{p}}$.

$\lambda_{i}^{j}=$ non negative constants associated each to $\mathbf{l}_{\mathbf{i}}^{\mathbf{j}}$.

$\mu=$ friction coefficient.

$\mathbf{w}_{i}^{j} \in \mathbb{R}^{6}=$ primitive contact wrench associated to the $j^{\text {th }}$ contact point $\mathbf{p}^{j}$.

$\phi_{i}^{j}=$ positive scalars associated each to $\mathbf{w}_{i}^{j}$.

$\mathbf{u}_{\mathbf{i}} \in \mathbb{R}^{6}=$ main direction $i$ of the task ellipsoid in the wrench space. 


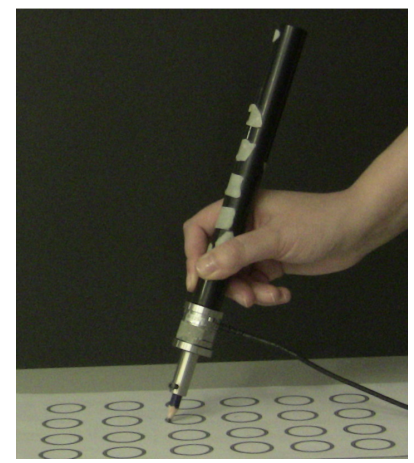

Figure 1: The sensorized tool is composed of a cylindrical handle to which is attached a 6D force/torque sensor. The end effector or the tool (pencil in this case) is then attached to the force/torque sensor enabling the human expert to accomplish a variety of tasks.

$\gamma_{i}=$ positive scalars associated with $\mathbf{u}_{\mathbf{i}}$.

\section{Modelling the Task}

To quantitatively model the TWS, capturing the wrenches exerted on the object while performing a task is essential. To capture these forces/torques applied on the object, we propose the usage of a sensorized tool that can be used by a human expert for this goal. Figure (1) illustrates the corresponding tool while being used during the task of drawing circles. It consists of a cylindrical handle to be grasped with, a $6 D$ force/torque sensor that can be attached to this handle and a tool or end effector attached to the force/torque sensor enabling the human expert to accomplish a variety of tasks such as writing, screw driving, cutting, etc. For performing this variety of tasks using this same tool, one needs only to replace the end effector with the one that corresponds to the task to perform.

This experimental setup allows a human expert to demonstrate a task during which forces/torques applied on the object can be collected and analysed. We have chosen to express this data in the object reference frame located at the center of the cylindrical handle. The black dots in figure (2) illustrate the forces and torques exerted on the object during the drawing circles task. This six dimensional data is then approximated by a $6 D$ ellipsoid. The main axes of this ellipsoid $\mathbf{u}_{\mathbf{i}} \in \mathbb{R}^{6}, i=1 . .6$, give the main directions in the wrench space that characterize the task and the associated length $\left\{\gamma_{i}>0\right\}, i=1 . .6$ give a measure of the range of the force/torque applied for the given task. Modeling this distribution is crucial to determine the positioning of the fingers that can generate the required range of force/torque for the task. For example, figure (2) shows the projection of the $6 D$ ellipsoid in the $3 D$ force and the $3 D$ torque domains. The main axis of each ellipsoid is represented in magenta. Notice that for the task of drawing circles, the forces applied on the $\mathrm{z}$ axis along the cylindrical handle is more important than the friction forces along the $x-y$ axis along the cross section of the handle. In the torque domain, as expected, the torques are mainly generated along the $\mathrm{x}$ and $\mathrm{y}$ axes, almost no torque was generated along the $\mathrm{z}$ axis. This figure also shows that the main force ellipsoid axis is perpendicular to the one of the torque 

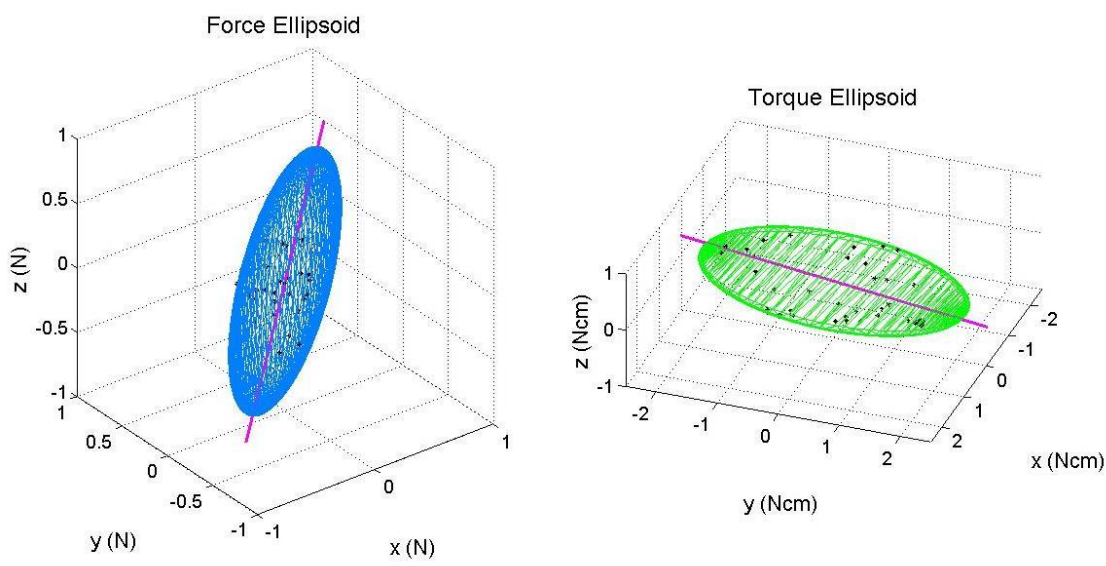

(a)

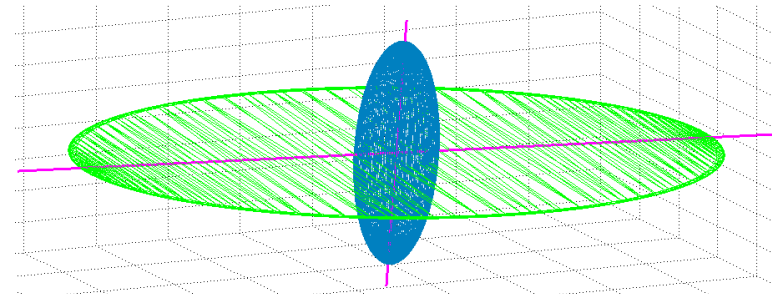

(b)

Figure 2: Drawing circles task: (a) the 6D ellipsoid is projected onto the 3D force domain (in blue) and onto the 3D torque domain (in green) The black dots are the collected force/torque data points. The main axis of each of the ellipsoids is represented in magenta; (b) the main force ellipsoid axis is perpendicular to the one of the torque ellipsoid.

ellipsoid. Thus, these task ellipsoids are capable of capturing the task characteristics and could be used for modelling the task requirements in terms of forces and torques.

\section{Human Hand Model}

We use for our study a human hand model as a proof of concept of the approach. The aim is to test whether the results of our optimization problem would correspond to grasps that are similar to those used by humans to accomplish a certain task.

\subsection{The Human hand kinematics}

The human hand kinematic model is inspired by the one used for the cyberglove $[8,7]$ and consists of 21 degrees of freedom distributed as follows: 4 revolute joints for modelling the index, middle, ring and pinky fingers (2 joints for flexion and abduction at the metacarpophalangeal junction (MCP) and one joint each at the proximal (PIP) and distal interphalangeal (DIP) junctions) and 5 revolute joints for modelling the thumb (the thumb requires one additional joint as it exhibits the ability for pronation/supination at the MCP junction). A detailed kinematic model of a 4 degrees of freedom finger is presented in paragraph (3.3). Figure 3 illustrates the revolute joints at each of the fingers. One should note that the distal link is coupled to the proximal one and it 


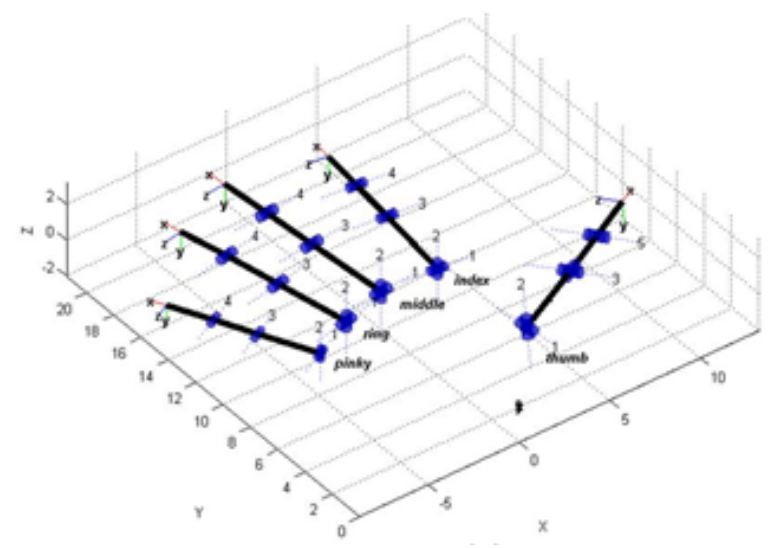

Figure 3: The Human hand kinematic model. Notice the 5 revolute joints of the thumb and the 4 revolute joints for each of the rest of the fingers.

moves with it at a fixed rate. For generating grasps later on in this paper, we use the thumb, index and middle fingers, resulting in 13 degrees of freedom.

\subsection{The Human hand force capabilities}

Similarly to the work in [17], we formalize in this paragraph the forces capabilities of a human finger. Figure (4) shows the tendons of a human index finger. A muscle or an actuator is attached to each tendon. Each muscle is assigned an activation level that varies from 0 indicating an inactive muscle to 1 at its maximum activation level. Following the biomechanical literature, the mapping from activation levels to joint torques is given through the following equation:

$$
\tau_{\text {max }}^{\mathbf{j}}=M^{j} P^{j} a^{j}
$$

Where $\tau_{\text {max }}^{\mathbf{j}}=\left\{\tau_{i \text { max }}^{j}\right\}^{1}$ and $\tau_{i \text { max }}^{j}$ stands for the maximal ith joint torque of the $j$ th finger (for the human hand $j$ varies between 1 and 5, for the index finger for example $i$ varies between 1 and 4$) ; \mathbf{a}^{\mathbf{j}}=\left\{a_{k}^{j}\right\}$ and $a_{k}^{j}$ stands for the activation level of the kth muscle of the $j$ th finger (the number of muscles per finger is equal to the number of tendons of that finger); $P^{j}$ is a $m^{j} \times m^{j}$ diagonal matrix, where $m^{j}$ is the number of muscles of the $j t h$ finger, $P^{j}$ converts activation levels to tendon forces, its diagonal element $p_{k k}$ corresponds to the maximum muscle force that can be supplied to tendon $k ; M^{j}$ is a $q^{j} \times m^{j}$ matrix, where $q^{j}$ is the number of joints of the $j^{\text {th }}$ finger, $M^{j}$ contains joint moment arm information and converts tendon forces to joint torques, (more details about $M^{j}, P^{j}$ and their corresponding values are given in annexe 10.1).

\subsection{Finger kinematics}

Figure 5 shows a detailed model of a 4 degrees of freedom model of the human hand finger. The revolute joints are represented as cylinders aligned with their axes, noted as $\mathbf{r}_{\mathbf{i}}^{\mathbf{j}}$. An orthonormal reference frame $R_{i}^{j}=\left(\mathbf{e}_{\mathbf{i}}^{\mathbf{j}}, v_{\mathbf{i}}^{\mathbf{j}}, \mathbf{r}_{\mathbf{i}}^{\mathbf{j}}\right)$ is attached to each joint, where $i=1, . ., q^{j}, j=1, . ., 5$ stand

\footnotetext{
${ }^{1}$ In the rest of this document, we use bold, normal and capital letters to represent respectively vectors, scalars and matrices.
} 

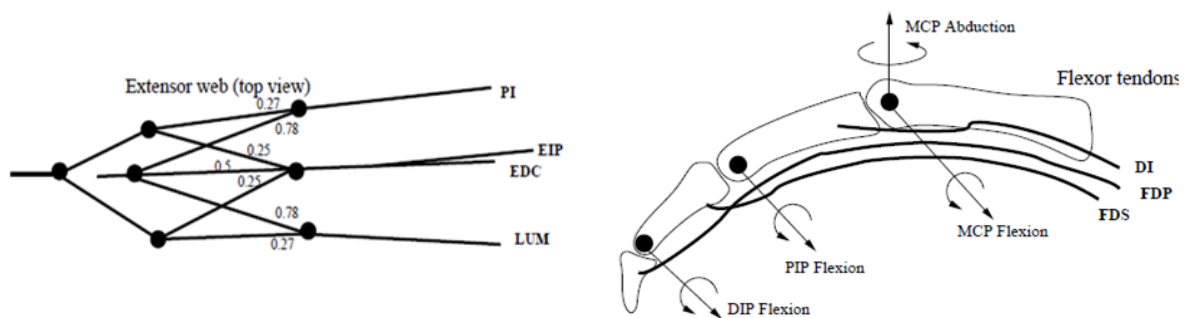

Figure 4: Flexor and extensor tendons of the human index finger. the numbers indicate ratios of outgoing forces at each branch point in the extensor web. Figure taken from [17].

respectively for the joint and finger numbers, $q^{j}$ is thus the number of DOFs of the $j^{\text {th }}$ finger. The $R_{i}^{j}$ are expressed according to an orthonormal reference frame, $R_{h}=\left(\mathbf{h}_{\mathbf{1}}, \mathbf{h}_{\mathbf{2}}, \mathbf{h}_{\mathbf{3}}\right)$, attached to the hand palm. $R_{h}$ is expressed in the object reference frame, $R_{O}=\left(\mathbf{o}_{1}, \mathbf{o}_{2}, \mathbf{o}_{3}\right)$, which is the origin of the system.

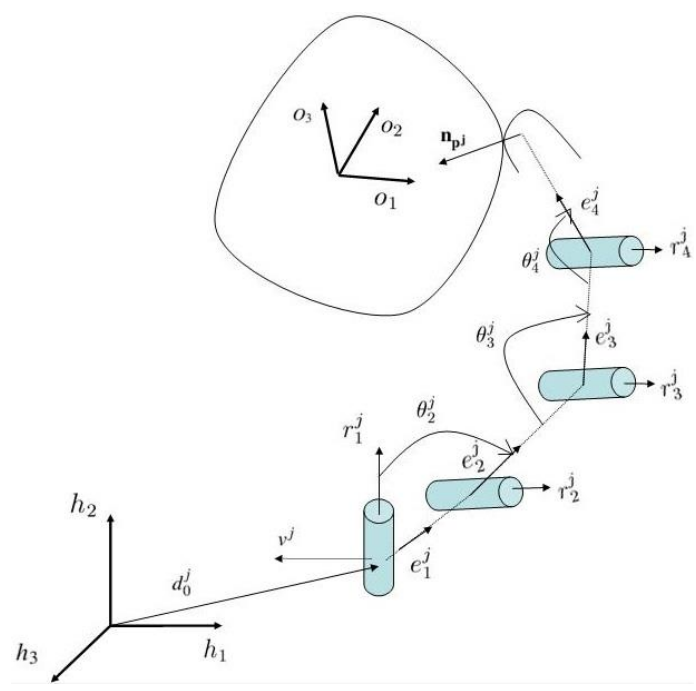

Figure 5: Kinematic structure of a human hand figer model, adapted from [19]. $R_{h}=\left(\mathbf{h}_{\mathbf{1}}, \mathbf{h}_{\mathbf{2}}, \mathbf{h}_{\mathbf{3}}\right)$ and $R_{o}=\left(\mathbf{o}_{\mathbf{1}}, \mathbf{o}_{2}, \mathbf{o}_{\mathbf{3}}\right)$ are the reference frames attached respectively to the hand palm and the object. The revolute joints are represented as cylinders aligned with their axes, noted as $r_{i}^{j}$. The $R_{i}^{j}=\left(e_{i}^{j}, v_{i}^{j}, r_{i}^{j}\right)$ are the reference frames attached to each joint. The $m_{i}^{j}$ vectors are not shown for simplicity on the display. $R_{1}^{j}$ represents the revolute joint responsible for the thumb opposition movement. $R_{2}^{j}, R_{3}^{j}$ and $R_{4}^{j}$ represent respectively the MP, PIP and DIP joints. $n_{p}^{j}$ is the normal vector at the contact point. $d_{0}^{j}$ is the finger anchor point defined relative to the hand reference frame. $\left\{\theta_{i}^{j}\right\}$ are the joint angles with $i=1, \ldots, 4$ in the thumb case. $v^{j}$ is a vector whose orientation is fixed relatively to the palm, it is useful for measuring $\theta_{1}^{j}$.

\section{Oject modeling}

We want to solve grasp synthesis as an optimization problem starting from a position of the hand away from the object and converging towards a good grasp. An implicit representation of 
the object surface is needed in order to test at each step of the optimization whether the fingers are inside, outside or placed on the object surface. Implicit surfaces are contours or isosurfaces, which can be described as the set of all the $x \in \mathscr{X} \subseteq \mathbb{R}^{3}$ for which the function $g: \mathscr{X} \rightarrow \mathbb{R}$ equals to zero. Thus $g$ gives a description of the object shape by telling for each location in space, $\mathbf{p}$, whether it is part or not of the object. A point $\mathbf{p}$ is defined by its $3 D$ cartesian coordinates $\left(p_{1}, p_{2}, p_{3}\right)$ :

$$
\begin{aligned}
g: \mathbb{R}^{3} \rightarrow \mathbb{R} ; g(\mathbf{p}) \quad & g\left(p_{1}, p_{2}, p_{3}\right) \\
= & \begin{cases}<0, & \mathbf{p} \text { is inside the object } \\
=0, & \mathbf{p} \text { is } \text { on the object surface } \\
>0, & \mathbf{p} \text { is outside the object }\end{cases}
\end{aligned}
$$

We use superquadrics to represent simple object shapes such as cylindrical ones and a probabilistic method, namely Gaussian Process Regression to represent complex object shapes as a single implicit function. For more details on this representation, please refer to our previous work [11].

\section{Constraints for generating task-oriented feasible grasps}

This paragraph details the different constraints required for the generation of optimal grasps according to a task related quality criterion. These constraints include the fingers placement on the object surface, the force/kinematic feasibility for the hand, the grasp stability, and collision avoidance requirements.

\subsection{Computing fingers locations}

We assume a frictional contact point model and thus three contacts are sufficient to achieve a stable grasp [14]. To solve the grasp synthesis problem, we have chosen to locate these contact points on the fingertips of the thumb, index and middle fingers.

If we select a contact point $\mathbf{p}^{\mathbf{j}}$ located on the fingertip of the $j^{\text {th }}$ finger, its coordinates in the object's frame of reference are obtained using the hand kinematic model described in section 3 :

$$
\mathbf{p}^{\mathbf{j}}=\mathbf{d}_{\mathbf{h}}+R_{h} \cdot \sum_{k=0}^{q^{j}} \mathbf{d}_{\mathbf{k}}^{\mathbf{j}}
$$

Where $\mathbf{d}_{\mathbf{h}}$ is the palm's position in the object's reference frame; $\mathbf{d}_{\mathbf{k}}^{\mathbf{j}}$ is the vector connecting two successive reference frames, $R_{k}^{j}$ and $R_{k+1}^{j}$, related to the $k^{\text {th }}$ and $(k+1)^{t h}$ links.

A grasp is obtained when $b$ contact points are established between the contact locations on the hand and the object's surface. Since we have at our disposal an analytical function $g: \mathbb{R}^{3} \rightarrow \mathbb{R}$ that describes the object's surface, see Section 4 , when a contact point $\mathbf{p}^{\mathbf{j}}$ located on the hand is in contact with the object, it satisfies:

$$
g\left(\mathbf{p}^{\mathbf{j}}\right)=0, \text { for all b contact points }
$$

Equation (3), if satisfied, guarantee that $b$ points located on the hand are in contact with the object's surface. However, this is not sufficient to ensure that the resulting grasp is feasible for the 
hand. An additional set of constraints is needed in order to take into account the range of motion of each of the joint angles. These constraints are directly derived from standard transformations across frames of reference and have been moved into appendix 10.2.

\subsection{Generating Force-closure grasps}

A grasp is said to be force-closure when the origin of its wrench space is contained inside the convex hull of the contact wrenches [15]. Computing force-closure grasps requires to compute the location of the contact points $\mathbf{p}^{\mathbf{j}}$ on the object surface as well as their corresponding contact normals $\mathbf{n}_{\mathbf{p}^{\mathbf{j}}}$, and is formulated as follows [15]:

$$
\begin{gathered}
\exists \phi_{i}^{j} \in \mathbb{R}, \phi_{i}^{j}>0, \quad \sum_{i, j} \phi_{i}^{j}=1, \quad j=1, . ., 5, \quad i=1, \ldots, l \\
\text { s.t } \quad \sum_{i, j} \phi_{i}^{j} \mathbf{w}_{i}^{j}=0
\end{gathered}
$$

Where $\phi_{i}^{j}$ are positive scalars ensuring that the primitive wrenches can positively span $\mathbb{R}^{6}$ and hence resist any external wrench; and $\mathbf{w}_{i}^{j} \in \mathbb{R}^{6}, i=1, . ., l$, is called a primitive contact wrench associated to the $j$ th contact point $\mathbf{p}^{j}$ and is computed as follows:

$$
\begin{array}{r}
\mathbf{w}_{i}^{j}=\left(\begin{array}{c}
\mathbf{l}_{i}^{j} \\
\mathbf{p}^{j} \times \mathbf{l}_{i}^{j}
\end{array}\right) \\
\mathbf{f}^{j}=\sum_{i=1}^{l} \lambda_{i}^{j} \mathbf{l}_{i}^{j}, \quad \lambda_{i}^{j} \geq 0
\end{array}
$$

$\mathbf{f}^{\mathbf{j}}$ is the force applied by the hand at a contact point $\mathbf{p}^{\mathbf{j}}$ and must satisfy Coulomb's law [9] and lie within the friction cone to prevent slippage along the surface of the object. Generally, the friction cone is linearized by a polyhedral convex cone with $l$ sides, where $\mathbf{l}_{\mathbf{i}}^{\mathbf{j}}$ represents the $i$ th edge vector of the polyhedral convex cone centered about the contact point internal normal $\mathbf{n}_{\mathbf{p}^{\mathbf{j}}}$. The coefficients $\lambda_{i}^{j}$ are non negative constants, see Appendix 10.3.

\subsection{Force feasibility criterion}

Following the biomechanical literature, we showed previously that the maximal $i^{\text {th }}$ joint torque of the $j^{t h}$ finger, $\tau_{i \text { max }}^{j}$ can be computed by equation (1). In order for the grasp to be force feasible, the hand in a grasp configuration needs to be able to apply on the object the wrenches required to accomplish the task without exceeding the limitations on its finger joint torques. Thus, we need to relate the wrenches applied on the object to the finger joint torques as follows [3]:

$$
\tau=J^{T} \mathbf{f}, \quad \text { and } \mathbf{w}=G \mathbf{f}
$$

Where $\tau \in \mathbb{R}^{q}$ is defined to group all the hand joint torques, $\tau=\left\{\tau_{i}^{j}\right\} ; q$ is the total number of joints and $\mathrm{b}$ is the number of fingers; the vector $\mathbf{f} \in \mathbb{R}^{3 b}$ groups all the applied forces at all the 
contact points; $J=\operatorname{diag}\left(J^{1}, J^{2}, . ., J^{b}\right)$ where $J^{j}$ is the jacobian matrix of the $j^{\text {th }}$ finger; $\mathbf{w} \in \mathbb{R}^{6}$ is the wrench applied on the object; $G \in \mathbb{R}^{6 \times 3 b}$ is the grasp matrix [12]. The equations (8) could be written as:

$$
\tau=J^{T}\left(G^{R} \mathbf{w}+A \mathbf{x}\right)
$$

Where $G^{R}$ is the right-inverse of the matrix $G$; $A$ is a basis of $\mathscr{N}(G)$ and $\mathbf{x}$ is a parameter vector. This formulation shows that wrench intensities with no effect on object motion are only those in $\mathscr{N}(G)$. However, it has also been shown that in order for all internal forces to be actively controlled by the joint actions, the null spaces of $G$ and $J$ need not to overlap and thus $\mathscr{N}(G) \cap$ $\mathscr{N}\left(J^{T}\right)=0,[20]$. This constraint could also be formulated as:

$$
J^{T} A \neq 0
$$

The limitation on the joint torques is expressed as:

$$
\tau \leq \tau_{\max }, \quad \text { with } \tau_{\max }=\left\{\tau_{i \max }^{j}\right\}, i=1 . . q^{j}, j=1 . . b
$$

\subsection{Task-oriented quality criterion}

In order to quantify the quality of a task-compatible grasp, we have chosen the generalized force manipulability criterion detailed in [3] that quantifies the ability of the grasp in ensuring the maximum transformation ratio between the hand joint torque and the object wrench space domains. This criterion is defined as:

$$
R_{w}=\frac{\mathbf{w}^{T} M_{w} \mathbf{w}}{\tau^{T} M_{\tau} \tau}
$$

Where $M_{w}$ and $M_{\tau}$ are symmetric positive definite weight matrices. The authors in [3] were interested in finding an optimum of this index or the maximum transformation ration with respect to both $\mathbf{w}$ and $\tau$. In our case, the task wrench space is well defined and thus $\mathbf{w}$ is fixed. Consequently, optimizing the index $R_{w}$ consists in minimizing $\tau^{T} M_{\tau} \tau$ in the direction of the task wrench space. Thus, our grasp quality criterion could be written as:

$$
\begin{gathered}
Q=\sum_{i=1}^{6} \gamma_{i} \tau_{\mathbf{u}_{\mathbf{i}}}{ }^{T} M_{\tau} \tau_{\mathbf{u}_{\mathbf{i}}} \\
\tau_{\mathbf{u}_{\mathbf{i}}}=J^{T}\left(G^{R} \mathbf{u}_{\mathbf{i}}+A \mathbf{x}\right)
\end{gathered}
$$

Where $\left\{\mathbf{u}_{\mathbf{i}}\right\}$ and $\left\{\gamma_{i}\right\}$, defined in section (2), are respectively the unitary vectors along the directions of the task wrench requirements and their corresponding normalized lengths. In this way, $\left\{\gamma_{i}\right\}$ factors weigh the relative importance of each force/torque direction for the accomplishment of the task. 


\subsection{Collision avoidance}

Our objects are described by an implicit surface function. This formulation allows to detect whether a point is inside, outside or on the surface of the object. Therefore, collision avoidance between the object and the hand is obtained by sampling several points $\mathbf{c}^{\mathbf{k}_{\mathbf{j}}}$, on the finger phalanges and palm; we compute the distance from these points to the object surface and add a new set of constraints to ensure all points are outside the object's surface:

$$
g\left(\mathbf{c}^{\mathbf{k}_{\mathbf{j}}}\right)>0, \forall k_{j}
$$

Where $k_{j}$ is the number of sampled points on the $j t h$ finger. Collision avoidance between the fingers is taken into account in the kinematic feasibility of the hand by considering the finger joint limits.

\section{Solving Optimal Grasp Synthesis as an optimization problem}

Given an analytical representation of the object and a hand kinematical model, this paragraph shows the formulation of grasp synthesis as a constrained optimization problem. Our objective function tries to maximize the task-oriented quality measure while satisfying force-closure constraints, hand kinematic constraints and collision constraints. This yields a constraint-based minimization for the set of parameters $\theta=\left\{\phi_{i}^{j}, i=1 . . m, j=1 . .3, R_{h}, \mathbf{d}_{\mathbf{h}}, R_{i}^{j}, i=1 . . q^{j}, j=1 . .3\right\}$, given by:

$$
\begin{gathered}
\underset{\theta}{\operatorname{argmin}} Q(\mathbf{p}) \\
\text { under the constraints }(3),(14),(4),(8-10),(16-23)
\end{gathered}
$$

While the objective function is convex, this is still a difficult optimization problem since the constraints are non-linear and the problem is high dimensional (in total 579 variables and 653 constraints). We have shown in our previous work [11] that Interior Point OPTimizer (IPOPT) method proposed by Wächter and Biegler [22] is adapted to solving problems with non linear constraints and thus we have chosen to apply this optimization technique on our new optimization problem. The algorithm is written in the Modeling Language for Mathematical Programming $(A M P L)$. By formulating the problem in $A M P L$, no analytical gradient computation is required. The IPOPT solver generates locally optimal solutions and we exploit this to generate multiple possible solutions for performing a task. This diversity in the possible grasps is a richness in manipulation capability that may prove useful in complex manipulation.

\section{Experimental Results}

The conducted experiments aimed at testing the ability of our approach of generating task compatible grasps. We first model the task using the data collected from the sensorized tool while a human demonstrates the task. Seven subjects participated in the experiment. The subjects were seated and were asked during the experiments to keep their elbow in the air and to rely as much as they can on the usage of the motion of their fingers for accomplishing the task, minimizing the usage of the wrist when it is possible. 

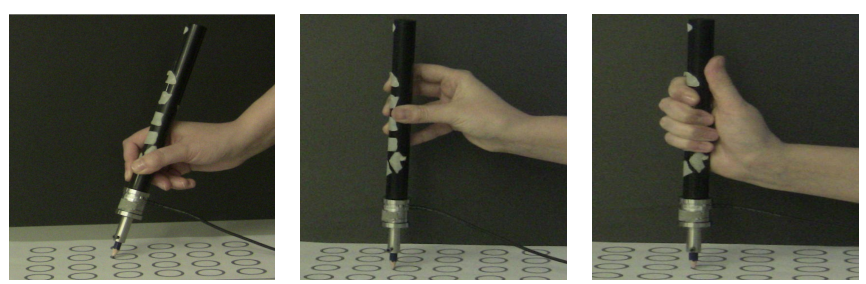

Figure 6: Three tested different grasps for performing the circles drawing task: tripod grasp, cylindrical grasp and power grasp.
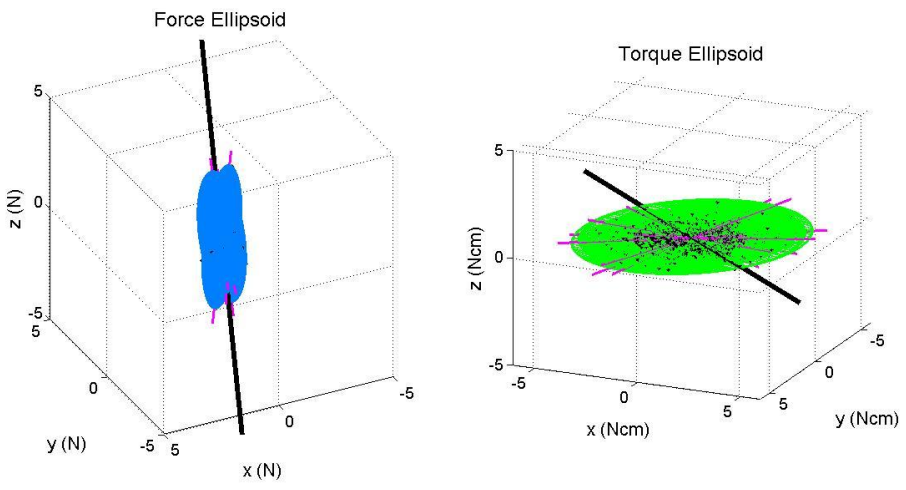

(a)
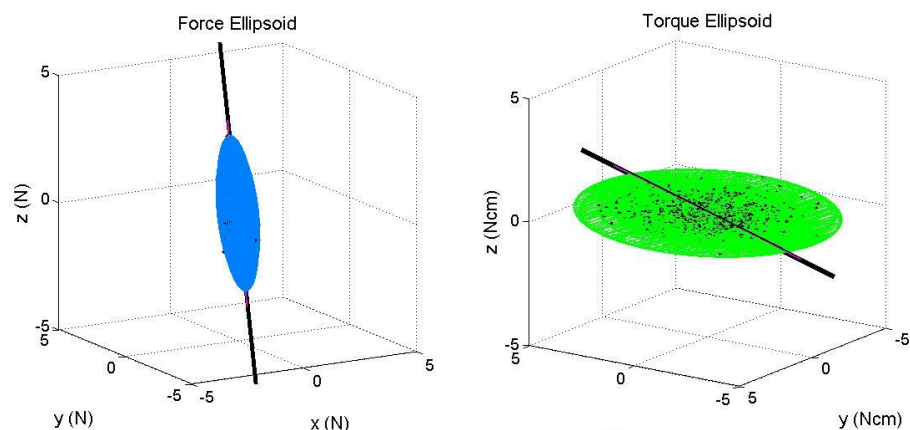

$\mathrm{x}(\mathrm{Ncm})$

(b)

Figure 7: Drawing circles task with a tripod grasp: (a) the force ellipsoids resulting from 21 trials are shown in blue; the torque ellipsoids are shown in green; the main axis of each of the ellipsoids is shown in magenta; the average main axis is shown in black in both force and torque domains. The black points correspond to the collected force/torque data; (b) the projection of the force/torque ellipsoids resulting from 21 trials along the average main axis represented in black. 


\subsection{Task Vs Grasp}

The aim of this experiment is to show whether the choice of the grasp influences the task description. For this purpose, one task is performed using different grasps and their models are compared.

\subsubsection{Experiment}

The task chosen for this experiment is the circles drawing task. The subjects were asked to use the sensorized tool as a pen and draw a circle by following a preprinted circular contour on a piece of a paper, repeating this motion 4 times. The choice of this task forces the subject to move the pen in the different directions that are involved in the motion of writing. The subjects were instructed to perform the circles drawing task using 3 different grasps of the sensorized tool, a tripod grasp, a cylindrical grasp and a power grasp, see figure(6). For each grasp type, each of the seven subjects performed the task three times for a total of 21 trials per grasp type. The $6 D$ force/torque data collected from each trial was expressed in the object reference frame located at the center of the tool's cylindrical handle and then approximated by a $6 D$ ellipsoid. In order to get a sense of how this ellipsoid is shaped in the object reference frame, we project this ellipsoid onto the $3 D$ force and $3 D$ torque domains. Figure (7.a) illustrates the 21 force ellipsoids as well as the 21 torque ellipsoids that correspond to the tripod grasp. The black dots represent the force/torque data collected along the circles drawing task. We plot in magenta the main axis along each of the $3 D$ ellipsoids in the force and torque domains. The average of these main axes is then represented as a black axis, showing the main directions in force and torque required by the task. Figure (7.b) shows the projection of these ellipsoids along these main directions. To get a sense of how their shapes vary from one to the other.

\subsubsection{Results}

In the following, we compute the axes lengths of the force/torque ellipsoids and check their variability across the 21 demonstrations. The following notation is used: $\mu_{f}$ and $\mu_{t}$ correspond to the average length of the 3 main axes of the force and torque ellipsoids respectively across the 21 trials; $\sigma_{f}$ and $\sigma_{t}$ are the corresponding standard deviations. $\mu_{f n}$ and $\mu_{t n}$ are the average length obtained after normalizing the axis length of each of the ellipsoid in order to get an insight about their shape and the importance of each axis in respective to the others; $\sigma_{f n}$ and $\sigma_{t n}$ are the corresponding standard deviations of the normalized shape. $\mu_{f n x y z}$ and $\mu_{t n-x y z}$ are the normalized average of the projection of the ellipsoids axes lengths along the $x, y, z$ axes of the object. $\sigma_{f} / \mu_{f}, \sigma_{f n} / \mu_{f n}$, $\sigma_{t} / \mu_{t}$ and $\sigma_{t n} / \mu_{t n}$ measure the different coefficients of variation and express the dispersion of the different examples as a percentage of their average. Tables $(1,2)$ show the force/torque ellipsoid shape of the circles drawing task for the 3 different grasp types, tables $(3,4)$ show the corresponding normalized force/torque ellipsoid shapes, and table (5) shows the normalized average of their projections along the $x, y, z$ axes of the object.

\subsubsection{Discussion}

By checking the force ellipsoid shapes in tables $(1,3,5)$ across the 3 different grasp types, we noticed the following: 
Table 1: Force ellipsoid shapes of the circles drawing task: average and standard deviation of the axes lengths of the force ellipsoids for the 3 grasp types, tripod, cylindrical and power grasps. Each line corresponds to the measures performed along one of the 3 axes of the ellipsoid, ordered according to their length ascending values.

\begin{tabular}{|c|c|c|c|c|c|c|c|c|}
\hline \multicolumn{10}{|c|}{ Force ellipsoid shapes } \\
\hline \multicolumn{3}{|c|}{ Tripod grasp } & \multicolumn{3}{c|}{ Cylindrical grasp } & \multicolumn{3}{c|}{ Power grasp } \\
\hline$\mu_{f}(\mathrm{~N})$ & $\sigma_{f}(N)$ & $\sigma_{f} / \mu_{f}$ & $\mu_{f}(\mathrm{~N})$ & $\sigma_{f}(N)$ & $\sigma_{f} / \mu_{f}$ & $\mu_{f}(\mathrm{~N})$ & $\sigma_{f}(N)$ & $\sigma_{f} / \mu_{f}$ \\
\hline 0.38 & 0.18 & 0.47 & 0.33 & 0.21 & 0.64 & 0.49 & 0.23 & 0.47 \\
\hline 0.52 & 0.23 & 0.44 & 0.42 & 0.24 & 0.57 & 0.66 & 0.32 & 0.48 \\
\hline 1.62 & 0.68 & 0.42 & 1.38 & 0.78 & 0.56 & 2.00 & 0.86 & 0.43 \\
\hline
\end{tabular}

Table 2: Torque ellipsoid shapes of the circles drawing task: average and standard deviation of the axes lengths of the torque ellipsoids for the 3 grasp types, tripod, cylindrical and power grasps. Each line corresponds to the measures performed along one of the 3 axes of the ellipsoid, ordered according to their length ascending values.

\begin{tabular}{|c|c|c|c|c|c|c|c|c|}
\hline \multicolumn{10}{|c|}{ Torque ellipsoid shapes } \\
\hline \multicolumn{3}{|c|}{ Tripod grasp } & \multicolumn{3}{c|}{ Cylindrical grasp } & \multicolumn{3}{c|}{ Power grasp } \\
\hline$\mu_{t}(\mathrm{~N})$ & $\sigma_{t}(N)$ & $\sigma_{t} / \mu_{t}$ & $\mu_{t}(\mathrm{~N})$ & $\sigma_{t}(N)$ & $\sigma_{t} / \mu_{t}$ & $\mu_{t}(\mathrm{~N})$ & $\sigma_{t}(N)$ & $\sigma_{t} / \mu_{t}$ \\
\hline 0.03 & 0.01 & 0.33 & 0.04 & 0.01 & 0.25 & 0.04 & 0.02 & 0.50 \\
\hline 2.63 & 1.22 & 0.46 & 2.19 & 1.31 & 0.60 & 3.18 & 1.52 & 0.48 \\
\hline 3.41 & 1.36 & 0.40 & 2.99 & 1.85 & 0.62 & 4.16 & 2.12 & 0.51 \\
\hline
\end{tabular}


- The shape of the force ellipsoids is similar for the 3 different grasp types, see the lengths of the 3 main axes of the ellipsoids in the different $\mu_{f n}$ columns, table (3). Notice that the dispersion among the different examples is also small and varies between 5 and $6 \%$ along the main axis and between 9 and $13 \%$ along the minor axes, see columns $\sigma_{f n} / \mu_{f n}$.

- Even though the shape of the ellipsoids is similar, their scale varies across trials (from 42 to $56 \%$ along the main axis, columns $\sigma_{f} / \mu_{f}$ ) and across grasp types (from $1.38 \mathrm{~N}$ to $2.0 \mathrm{~N}$ along the main axis, columns $\mu_{f}$ ), table (1). Notice that the forces applied on the object during a power grasp are higher than the ones involving a tripod or a cylindrical grasp. This is probably due to the fact that the subjects tend to press more on the paper when they're holding the pen using a power grasp.

- According to columns $\mu_{f n-x y z}$, the major force applied on the object is, as expected, directed along the $z$ axis of the tool, table (5). The forces along the $x$ and $y$ axes correspond to the friction forces along both directions. They are very close and this is due to the circular motion of the pen involving friction in both directions.

Similarly, if we check the values in tables $(2,4,5)$ related to the torque ellipsoids across the 3 different grasp types, we notice the following:

- The shape of the torque ellipsoids is similar for the 3 different grasp types, see the lengths of the 3 main axes of the ellipsoids in the different $\mu_{t n}$ columns, table (4). Notice that the dispersion among the different examples is also small and varies between 5 and $9 \%$ along the 2 major axes and between 30 and $60 \%$ along the minor axes, see columns $\sigma_{t n} / \mu_{t n}$. This big variation along the minor axis is due to the fact that the average normalized torque value along this axis is around 0.01N.cm, almost non existent and the variance is due to noise.

- Even though the shape of the ellipsoids is similar, their scale varies across trials (columns $\sigma_{t} / \mu_{t}$ ) and across grasp types (columns $\mu_{t}$ ), table (2). Notice that the torques applied on the object during a power grasp are higher than the ones involving a tripod or a cylindrical grasp.

- According to columns $\mu_{t n-x y z}$, the 2 major torques applied on the object are, as expected, directed along the $x$ and $y$ axes of the tool, table (5). The torques along the $z$ axes are almost non existent.

This experiment shows that the scale of the task ellipsoids may vary across trials and grasp types but their shape remains very similar with a dispersion between 5 and $9 \%$ along force/torque requirements. This variation is probably related to how well each subject has performed the task. For example, some subjects took the time to guide the pen precisely around the preprinted circles while others where less precise in their motions. Additionally to this dispersion measurement, we compute in the following section another metric showing the statistical similarity of these ellipsoids. 
Table 3: Normalized force ellipsoid shapes of the circles drawing task: normalized average and standard deviation of the axes lengths of the force ellipsoids for the 3 grasp types, tripod, cylindrical and power grasps. Each line corresponds to the measures performed along one of the 3 axes of the ellipsoid, ordered according to their length ascending values.

\begin{tabular}{|c|c|c|c|c|c|c|c|c|}
\hline \multicolumn{8}{|c|}{ Normalized force ellipsoid shapes } \\
\hline \multicolumn{3}{|c|}{ Tripod grasp } & \multicolumn{3}{c|}{ Cylindrical grasp } & \multicolumn{3}{c|}{ Power grasp } \\
\hline$\mu_{f n}(\mathrm{~N})$ & $\sigma_{f n}(N)$ & $\sigma_{f n} / \mu_{f n}$ & $\mu_{f n}(\mathrm{~N})$ & $\sigma_{f n}(N)$ & $\sigma_{f n} / \mu_{f n}$ & $\mu_{f n}(\mathrm{~N})$ & $\sigma_{f n}(N)$ & $\sigma_{f n} / \mu_{f n}$ \\
\hline 0.15 & 0.02 & 0.13 & 0.15 & 0.02 & 0.13 & 0.15 & 0.02 & 0.13 \\
\hline 0.20 & 0.02 & 0.10 & 0.20 & 0.02 & 0.10 & 0.21 & 0.02 & 0.09 \\
\hline 0.65 & 0.03 & 0.05 & 0.65 & 0.04 & 0.06 & 0.64 & 0.035 & 0.05 \\
\hline
\end{tabular}

Table 4: Normalized torque ellipsoid shapes of the circles drawing task: normalized average and standard deviation of the axes lengths of the torque ellipsoids for the 3 grasp types, tripod, cylindrical and power grasps. Each line corresponds to the measures performed along one of the 3 axes of the ellipsoid, ordered according to their length ascending values.

\begin{tabular}{|c|c|c|c|c|c|c|c|c|}
\hline \multicolumn{10}{|c|}{ Normalized torque ellipsoid shapes } \\
\hline \multicolumn{4}{|c|}{ Tripod grasp } & \multicolumn{3}{c|}{ Cylindrical grasp } & \multicolumn{3}{c|}{ Power grasp } \\
\hline$\mu_{t n}(\mathrm{~N})$ & $\sigma_{t n}(N)$ & $\sigma_{t n} / \mu_{t n}$ & $\mu_{t n}(\mathrm{~N})$ & $\sigma_{t n}(N)$ & $\sigma_{t n} / \mu_{t n}$ & $\mu_{t n}(\mathrm{~N})$ & $\sigma_{t n}(N)$ & $\sigma_{t n} / \mu_{t n}$ \\
\hline 0.01 & 0.003 & 0.30 & 0.01 & 0.006 & 0.60 & 0.007 & 0.003 & 0.43 \\
\hline 0.42 & 0.04 & 0.09 & 0.42 & 0.041 & 0.09 & 0.43 & 0.03 & 0.07 \\
\hline 0.57 & 0.04 & 0.07 & 0.57 & 0.04 & 0.07 & 0.56 & 0.03 & 0.05 \\
\hline
\end{tabular}

Table 5: Circles drawing task: the normalized average of the projection of the force/torque ellipsoid axes lengths along the $x, y, z$ axes of the object for the 3 grasp types, tripod, cylindrical and power grasps. The 3 rows illustrate respectively the ellipsoid lengths along the $x, y$ and $z$ axes of the object reference frame.

\begin{tabular}{|c|c|c|c|c|c|}
\hline \multicolumn{2}{|c|}{ Tripod grasp } & \multicolumn{2}{c|}{ Cylindrical grasp } & \multicolumn{2}{c|}{ Power grasp } \\
\hline$\mu_{f n \_x y z}(N)$ & $\mu_{t n \_x y z}(N . c m)$ & $\mu_{f n_{-x y z}(N)}$ & $\mu_{t n \_x y z}(N . c m)$ & $\mu_{f n_{-x y z}}(N)$ & $\mu_{t n \_x z z}(N . c m)$ \\
\hline 0.19 & 0.49 & 0.19 & 0.55 & 0.18 & 0.50 \\
\hline 0.22 & 0.50 & 0.18 & 0.44 & 0.19 & 0.49 \\
\hline 0.59 & 0.01 & 0.63 & 0.01 & 0.63 & 0.01 \\
\hline
\end{tabular}



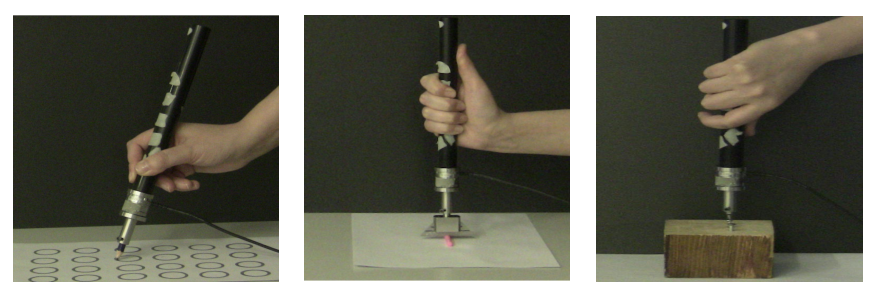

Figure 8: Three different tasks were performed with the sensorized tool: circles drawing, cutting and screw driving.

\subsection{Modelling different tasks}

The aim of this experiment is to show that the task ellipsoids are able to capture the differences in terms of force/torque requirements across different tasks. Three tasks were chosen for this purpose, each demanding different force and torque requirements. Since the task model does not depend on the choice of grasp, in the following, the subjects have selected a grasp of their choice to perform the required task.

\subsubsection{Experiment}

Three tasks were analysed: drawing circles, cutting and using a screw driver, see figure (8):

- Circles drawing task: similar description to previous section.

- Cutting task: the pen end effector was replaced for this task by a blade and the subjects were asked to use the sensorized tool as a knife, moving the blade back and forth until they cut a small piece of candle. While the writing/drawing task involves mainly forces along the cylindrical handle of the tool, the cutting task involves forces along both directions, the cylindrical handle and the cutting direction.

- Screw driving task: this task was chosen because it involves not only applying forces along the cylindrical handle of the tool but also requires applying a torque along the same direction. During this task, the end effector of the tool was replaced by a screw driver tip, the screws had a black mark and the subjects were asked to drive the screws in a piece of wood until the black mark was reached.

A task needed to be completed successfully by the subject in order to take the corresponding data into account. Again, seven subjects performed successfully each of the previous tasks 3 times, for a total of 21 trials per task. Tables $(6,6)$ show the force/torque ellipsoid shapes of the circles drawing, cutting and screw driving tasks, tables $(8,9)$ show the corresponding normalized force/torque ellipsoid shapes, and table (10) shows the normalized average of their projections along the $x, y, z$ axes of the object.

\subsubsection{Results}

Figure (10) illustrates the force and torque ellipsoids of the three tasks across the different trials. In the previous section, we have already showed how the shape of the circles drawing task ellipsoids corresponded to our intuition about the task requirements. If we consider now the task of cutting, we notice in figure (10.b) that the forces applied on the object are mainly along the $x$ and 


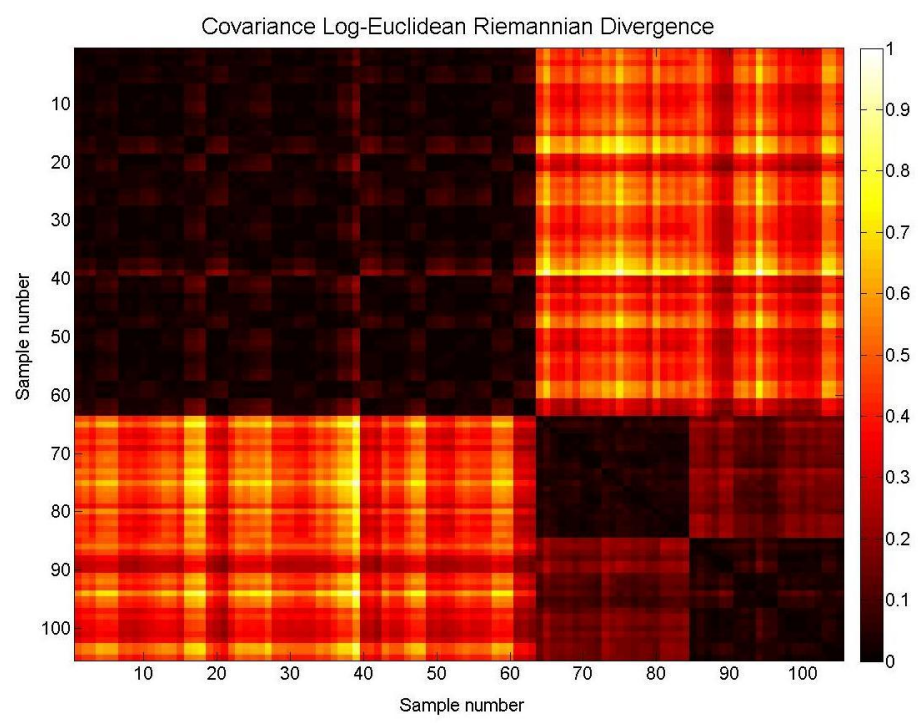

Figure 9: The normalised Riemannian metric applied on our data set. The first 63 data points correspond to the $6 D$ ellipsoids resulting from the circles drawing experiment using the 3 different grasp types (21 samples per grasp type). The following 21 samples correspond to the cutting experiment data. The last 21 data points correspond to the screw driving experiment, resulting in a total of 105 ellipsoids.

$z$ axes which are the directions of the blade and the cylindrical handle respectively. The torques related to this task are mainly along the $y$ axis and they are due to the back and forth motion cutting motion along the $x$ axis. As for the screw driving task, we notice in figure (10.c) that the forces are mainly along the $z$ axis. The torques involved in this task are as well important along the $z$ axis but they also have a considerable value along the $x$ and $y$ axes, see column $\mu_{t n}$ in table (10). One would expect for the screw driving task to have mainly torques along the $z$ axis only but in practice when the subjects were trying to drive the screw within the wooden block, the screw driver tip was not immediately directed towards the center of the screw, it was often touching the edges of the screw, resulting in generating torques along the $x$ and $y$ directions. Thus, these task ellipsoids managed to capture what the subject was really doing. Notice also the columns $\sigma_{f n} / \mu_{f n}$ and $\sigma_{t n} / \mu_{t n}$ in tables $(8,9)$ showing once more time that the shape of the ellipsoids per task are similar across trials with a dispersion varying between 5 and $12 \%$ along the main axes defining the force requirements and between 4 and 12\% along the main axes defining the torque requirements, with the exception of the screw driving task where a torque was exerted along the 3 axes and one of them showed a dispersion of $28 \%$. While the subjects performed the circles drawing and cutting easily, they were not experts in performing screw driving tasks and as explained previously, they were clumsy in the way they directed the tool to fit within the screw resulting in this big dispersion along the screw driving torque task ellipsoid. The range of force/torque involved in these experiments, could easily be checked from the columns $\mu_{f}$ and $\mu_{t}$ in tables $(6,7)$ showing that the average forces involved in the circles drawing, cutting and screw driving tasks along the major axis are respectively $1.67 N$, $8.3 \mathrm{~N}$ and $11.49 \mathrm{~N}$ while the corresponding torques are of $3.52 \mathrm{~N} . \mathrm{cm}, 61.75 \mathrm{~N} . \mathrm{cm}$ and $9.64 \mathrm{~N} . \mathrm{cm}$.

In order to quantify the similarity and dissimilarity between the task ellipsoids, we need a metric measuring the distance between two ellipsoids. An ellipsoid belongs to a special category 
Table 6: Force ellipsoid shapes of the circles drawing, cutting and screw driving tasks: normalized average and standard deviation of the axes lengths of the force ellipsoids for the 3 tasks. Each line corresponds to the measures performed along one of the 3 axes of the ellipsoid, ordered according to their length ascending values.

\begin{tabular}{|c|c|c|c|c|c|c|c|c|}
\hline \multicolumn{10}{|c|}{ Force ellipsoid shapes } \\
\hline \multicolumn{3}{|c|}{ Drawing task } & \multicolumn{3}{c|}{ Cutting task } & \multicolumn{3}{c|}{ Screw driving task } \\
\hline$\mu_{f}(\mathrm{~N})$ & $\sigma_{f}(N)$ & $\sigma_{f} / \mu_{f}$ & $\mu_{f}(\mathrm{~N})$ & $\sigma_{f}(N)$ & $\sigma_{f} / \mu_{f}$ & $\mu_{f}(\mathrm{~N})$ & $\sigma_{f}(N)$ & $\sigma_{f} / \mu_{f}$ \\
\hline 0.38 & 0.18 & 0.47 & 0.97 & 0.33 & 0.34 & 1.00 & 0.57 & 0.57 \\
\hline 0.52 & 0.23 & 0.44 & 4.82 & 1.21 & 0.25 & 1.57 & 1.00 & 0.64 \\
\hline 1.62 & 0.68 & 0.42 & 8.33 & 1.61 & 0.19 & 11.49 & 3.01 & 0.26 \\
\hline
\end{tabular}

of symmetric matrices, which is the positive definite symmetric matrices, see equation (??), and these matrices form a Riemannian manifold. Ordinary Euclidean distance ignores such a manifold structure, therefore, in the literature, a Riemannian metric is used to compute the distance between these matrices [2]. This metric maps the symmetric positive definite matrices to a flat Riemannian space so the ordinary Euclidean distance could be used, and is defined as:

$$
D(X, Y)=-\|\log (X)-\log (Y)\|_{F}
$$

Where $\|.\|_{F}$ stands for the Frobenius norm. When normalizing this metric by the maximal distance in the data set, a distance of 0 would correspond to the case where both ellipsoids have identical shapes and 1 corresponds to the case where two ellipsoids have the most different shapes. Figure (9) illustrates the different values of the normalized Riemannian metric when applied to our data set. The first 63 data points correspond to the $6 D$ ellipsoids resulting from the circles drawing experiment using the 3 different grasp types ( 21 samples per grasp type). The following 21 samples correspond to the cutting experiment data. The last 21 data points correspond to the screw driving experiment, resulting in a total of 105 ellipsoids. We can easily distinguish in this figure 3 different groups of ellipsoids corresponding to the 3 different tasks, which shows that the task ellipsoids managed to capture the differences in the task requirements. We can also notice that the 3 different drawing grasp types are similar to each others.

\subsection{Grasp Synthesis}

The experiments presented in this paragraph aimed at testing the optimization algorithm's ability to generate for a given task, grasps that are feasible for the hand's kinematics and that are adapted to the task requirements. Since we model the task from human demonstration, we have chosen to test our algorithm on a human hand kinematic model in order to check whether the obtained grasps could be similar to those chosen by a human subject. The sensorized tool is described in the optimization algorithm by a cylindrical superquadric with length of $20 \mathrm{~cm}$ and a diameter of $2 \mathrm{~cm}$. To explore the diversity of possible solutions, we run the algorithm starting from different initial conditions. We generate 42 orientations of the palm that span uniformly 

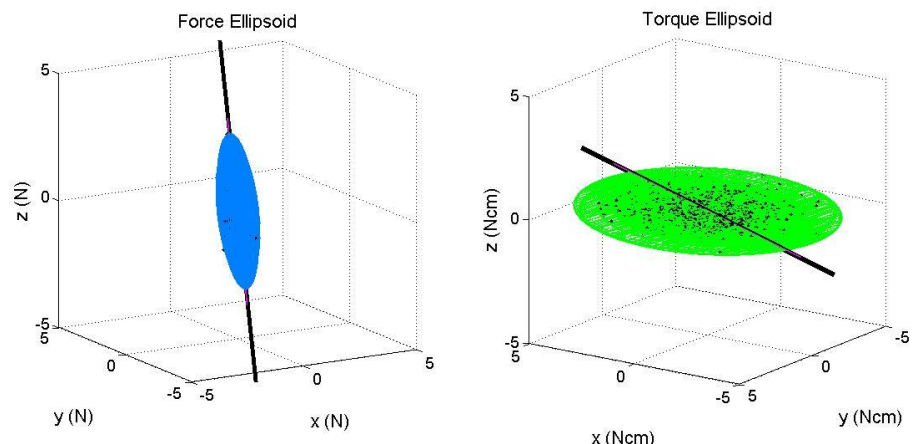

(a)
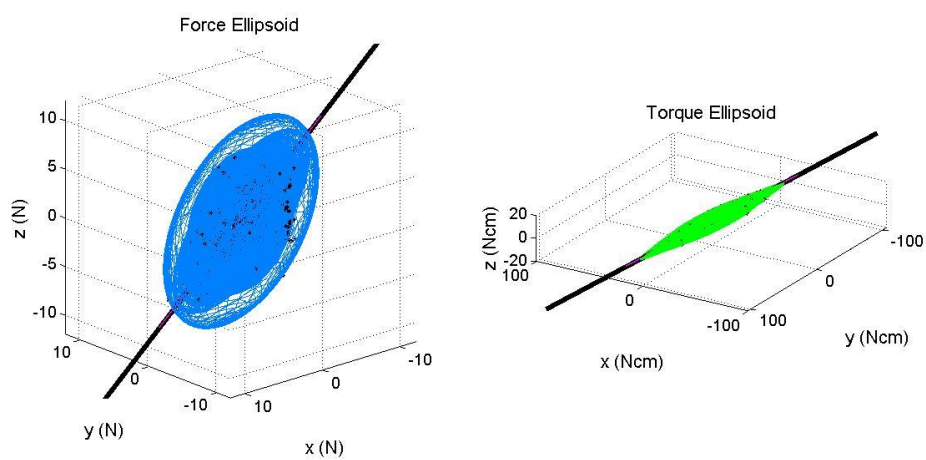

(b)
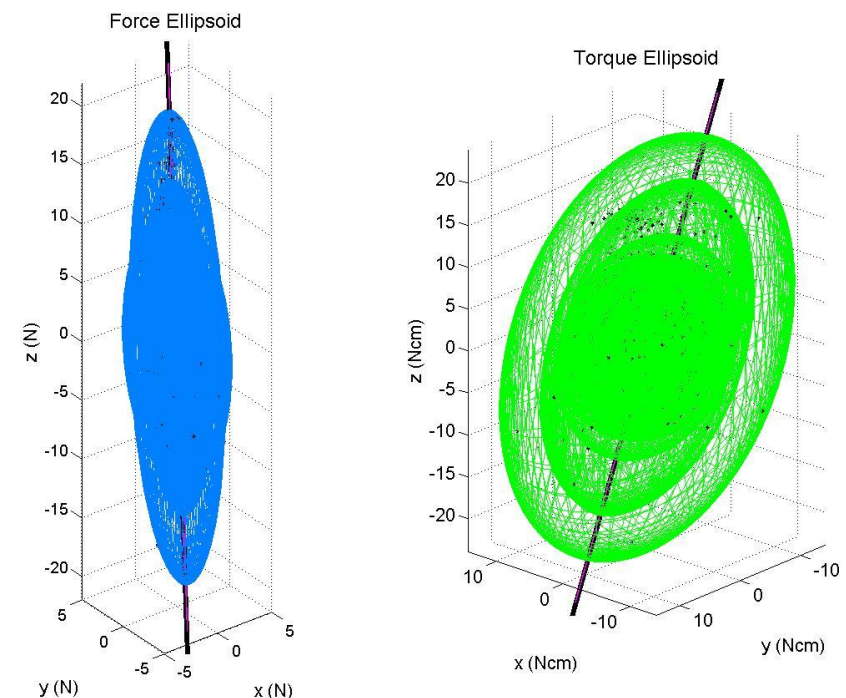

(c)

Figure 10: Force ellipsoids in blue and torque ellipsoids in green corresponding to the tasks of writing (a), cutting (b) and screw driving (c). 
Table 7: Torque ellipsoid shapes of the circles drawing, cutting and screw driving tasks: normalized average and standard deviation of the axes lengths of the torque ellipsoids for the 3 tasks. Each line corresponds to the measures performed along one of the 3 axes of the ellipsoid, ordered according to their length ascending values.

\begin{tabular}{|c|c|c|c|c|c|c|c|c|}
\hline \multicolumn{10}{|c|}{ Torque ellipsoid shapes } \\
\hline \multicolumn{3}{|c|}{ Drawing task } & \multicolumn{3}{c|}{ Cutting task } & \multicolumn{3}{c|}{ Screw driving task } \\
\hline$\mu_{t}(\mathrm{~N})$ & $\sigma_{t}(N)$ & $\sigma_{t} / \mu_{t}$ & $\mu_{t}(\mathrm{~N})$ & $\sigma_{t}(N)$ & $\sigma_{t} / \mu_{t}$ & $\mu_{t}(\mathrm{~N})$ & $\sigma_{t}(N)$ & $\sigma_{t} / \mu_{t}$ \\
\hline 0.03 & 0.01 & 0.33 & 2.09 & 0.85 & 0.41 & 3.15 & 1.16 & 0.37 \\
\hline 2.63 & 1.22 & 0.46 & 8.30 & 3.00 & 0.36 & 6.50 & 3.29 & 0.51 \\
\hline 3.41 & 1.36 & 0.40 & 61.75 & 12.98 & 0.21 & 9.64 & 6.13 & 0.64 \\
\hline
\end{tabular}

Table 8: Normalized force ellipsoid shapes of the circles drawing, cutting and screw driving tasks: normalized average and standard deviation of the axes lengths of the force ellipsoids for the 3 tasks. Each line corresponds to the measures performed along one of the 3 axes of the ellipsoid, ordered according to their length ascending values.

\begin{tabular}{|c|c|c|c|c|c|c|c|c|}
\hline \multicolumn{8}{|c|}{ Normalized force ellipsoid shapes } \\
\hline \multicolumn{3}{|c|}{ Drawing task } & \multicolumn{3}{c|}{ Cutting task } & \multicolumn{3}{c|}{ Screw driving task } \\
\hline$\mu_{f n}(\mathrm{~N})$ & $\sigma_{f n}(N)$ & $\sigma_{f n} / \mu_{f n}$ & $\mu_{f n}(\mathrm{~N})$ & $\sigma_{f n}(N)$ & $\sigma_{f n} / \mu_{f n}$ & $\mu_{f n}(\mathrm{~N})$ & $\sigma_{f n}(N)$ & $\sigma_{f n} / \mu_{f n}$ \\
\hline 0.15 & 0.02 & 0.13 & 0.07 & 0.02 & 0.29 & 0.07 & 0.02 & 0.28 \\
\hline 0.20 & 0.02 & 0.10 & 0.34 & 0.04 & 0.12 & 0.11 & 0.04 & 0.36 \\
\hline 0.65 & 0.03 & 0.05 & 0.59 & 0.06 & 0.10 & 0.82 & 0.06 & 0.07 \\
\hline
\end{tabular}

Table 9: Normalized torque ellipsoid shapes of the circles drawing, cutting and screw driving tasks: normalized average and standard deviation of the axes lengths of the force ellipsoids for the 3 tasks. Each line corresponds to the measures performed along one of the 3 axes of the ellipsoid, ordered according to their length ascending values.

\begin{tabular}{|c|c|c|c|c|c|c|c|c|}
\hline \multicolumn{8}{|c|}{ Normalized torque ellipsoid shapes } \\
\hline \multicolumn{4}{|c|}{ Drawing task } & \multicolumn{3}{c|}{ Cutting task } & \multicolumn{3}{c|}{ Screw driving task } \\
\hline$\mu_{t n}(\mathrm{~N})$ & $\sigma_{t n}(N)$ & $\sigma_{t n} / \mu_{t n}$ & $\mu_{t n}(\mathrm{~N})$ & $\sigma_{t n}(N)$ & $\sigma_{t n} / \mu_{t n}$ & $\mu_{t n}(\mathrm{~N})$ & $\sigma_{t n}(N)$ & $\sigma_{t n} / \mu_{t n}$ \\
\hline 0.01 & 0.003 & 0.30 & 0.03 & 0.01 & 0.33 & 0.18 & 0.05 & 0.28 \\
\hline 0.42 & 0.04 & 0.09 & 0.12 & 0.04 & 0.33 & 0.34 & 0.04 & 0.12 \\
\hline 0.57 & 0.04 & 0.07 & 0.85 & 0.04 & 0.04 & 0.48 & 0.06 & 0.12 \\
\hline
\end{tabular}


Table 10: Drawing, cutting and screw driving tasks: the normalized average of the projection of the force/torque ellipsoid axes lengths along the $x, y, z$ axes of the object for the 3 different tasks. The 3 rows illustrate respectively the ellipsoid lengths along the $x, y$ and $z$ axes of the object reference frame.

\begin{tabular}{|c|c|c|c|c|c|}
\hline \multicolumn{2}{|c|}{ Drawing task } & \multicolumn{2}{c|}{ Cutting task } & \multicolumn{2}{c|}{ Screw driving task } \\
\hline$\mu_{f n \_x y z}(N)$ & $\mu_{t n_{-x y z}}(N . c m)$ & $\mu_{f n \_x y z}(N)$ & $\mu_{t n \_x y z}(N . c m)$ & $\mu_{f n \_x y z}(N)$ & $\mu_{t n \_x y z}(N . c m)$ \\
\hline 0.19 & 0.49 & 0.44 & 0.19 & 0.10 & 0.37 \\
\hline 0.22 & 0.50 & 0.09 & 0.76 & 0.10 & 0.36 \\
\hline 0.59 & 0.01 & 0.46 & 0.05 & 0.80 & 0.27 \\
\hline
\end{tabular}

\begin{tabular}{|l|c|c|}
\hline Quality & 0.37 & 9.7 \\
\hline & & \\
\hline
\end{tabular}

Table 11: The grasp quality of the generated grasps for the circles drawing task.

the different directions in space, initialized from 3 different locations (the palm is positioned on the diagonal of the reference system attached to the center of the object, the palm aligned with the middle of the object height and the palm placed above the object), for a total of 126 different initial hand postures. Starting from each initial configuration, we computed grasps on the cylindrical tool for the 3 different task models, writing, cutting and screw driving. The experiments were run on an AMD Opteron machine with $47 \mathrm{~GB}$ and a $\mathrm{CPU}$ at $2.4 \mathrm{GHz}$. For the 126 trials, the algorithm converges to 7 locally optimal solutions in the case of circles drawing task, 20 solutions in the case of the cutting task and 3 in the case of the screw driving task. Many of these were similar in terms of hand/fingers configurations. Tables $(11,12$ and figure 11) illustrate the different configurations obtained for each of the tasks and show their corresponding quality. The average computation time to find a solution was of $459 \mathrm{sec}$ with a standard deviation of $186 \mathrm{sec}$. The solutions were plotted using the SynGrasp Matlab toolbox for grasp analysis [13].

\subsubsection{Discussion}

The grasps chosen by the subjects to perform the writing, cutting and screw driving tasks often involved 4 contact points or more and were distributed on different parts involving many times the sides of the fingers. Consequently, the interpretation of the computed grasps from a humanlikeness point of view is not intuitive. However, our choice of choosing fingertip grasp generation was mainly due to the fact that current robotic hands are still far from reaching the dexterity of a human hand. For example, the Barrett hand has three fingers and only one degree of freedom 


\begin{tabular}{|l|c|c|c|}
\hline Quality & 0.04 & 0.35 & 2.7 \\
\hline & & &
\end{tabular}

Table 12: The grasp quality of the generated grasps for the cutting task.

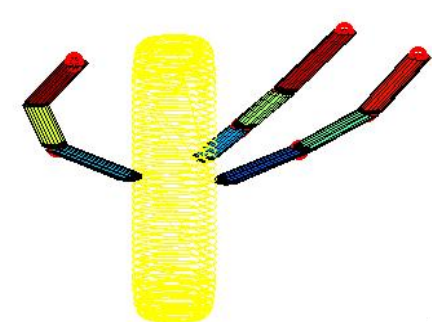

Figure 11: The generated grasp for the screw driving task.

per finger and consequently it is kinematically limited in the sense that one finger is not able to touch the side of another finger similarly to a human hand. To prove that our approach is able to be directly transferable to any robotic hand model and since the minimum required number for grasp stability considering frictional contact points is 3 [14], we have chosen to initially test our algorithm on the human hand model considering only 3 contact points on the thumb, index and middle fingers resulting in 13 degrees of freedom. This shows the ability of the algorithm to generate task-oriented grasps for any hand whether simple or complex in terms of number of degrees of freedom. If one is then interested in generating grasps that involve more contact points, one needs to sample possible contact points on the hand and reformulate the problem as a discrete optimization problem varying both the number and the locations of the contact points.

Concerning the choice of a sensorized tool to model the task, one could also think of collecting task oriented data in a similar way to the paper of Kry et al. [10] where the authors collected their data by either placing pressure sensors on the fingertips of the user or by placing a force/torque sensor underneath the object to measure interaction forces. While placing a force/torque sensor underneath the paper the user will be writing on is simple and allows the hand to perform a natural writing motion with a standard writing tool, sensorizing the environment in the case of the screw driving or the cutting task is more tricky and requires a different setup for each of the tasks. Placing pressure sensors on the fingertips could be a solution that is adapted for measuring interaction forces across several manipulation tasks but first needs to be coupled with an estimation of the torques applied on the object through tracking its motion and second it restricts the user to the usage of his/her fingertips while manipulating the object which might not be their natural way of 
handling the object. Using a sensorized tool is useful in the sense that, with the same setup, one can capture interaction forces/torques across several tasks. In this case, the tool structure might be slightly different from the usual knife or pen or screw driver and thus capturing the task model is also not done in a very natural way. However, no matter the way adopted to capture the interaction forces/torques, it does not affect the core of our approach which is to model the task constraints from user demonstration and include them in the formulation of the grasp synthesis approach.

If we consider the solutions generated with our task-oriented grasp synthesis algorithm, we notice that different optimal grasps were obtained for different task descriptions. The only solution that was found in the case of the screw driving task positions the hand in a way to have the fingers holding the cylindrical tool around its central part. This configuration allows the hand to exert both forces and torques along the $z$-axis which is the main axis of the cylindrical handle, allowing the hand to accomplish the task requirements. Notice that this configuration was of low quality for the cutting task as it requires to apply a force along the $x$-axis and a torque along the $y$-axis requiring the palm to be placed more parallel to the side of the tool instead of being perpendicular to its axis. Finally, the grasps obtained in the case of the circles drawing task are intuitive and resemble the usual tripod grasp used for writing. If we compare these results to our previous work in [11], where the objective function was to minimize the norm of the joint torque vector of the hand ensuring grasp stability and where no task constraints were included in that formulation, we notice that the solutions obtained were a variety of force-closure grasps, which are not necessarily task-oriented. In this paper, the usage of the task constraints and task-oriented objective function forces the optimization algorithm to converge towards a task-oriented grasp. The results show that different solutions were obtained for different tasks, while the solutions obtained in [11] were a set of generic force-closure grasps. Thus, one could conclude that including these task-oriented constraints and their corresponding objective function resulted indeed in task-oriented grasps.

\section{Conclusion}

This paper presented a detailed approach for task modelling through human demonstration and through the usage of a sensorized tool. It also showed that once a task $6 D$ model is obtained, its main axes in the force/torque domain as well their relative importance could be identified and plugged into a task-oriented quality criterion. This quality measure was used as an objective function in the formulation of grasp synthesis as an optimization problem generating grasps that are feasible for the hand kinematic model and optimal according to the task requirements. Our approach was tested on a human-like hand model and is directly transferable for any robotic hand model.

\section{Acknowledgments}

We would like to thank François Margot for advices on efficiently using IPOPT to solve our problem and for giving us access to the computing facilities at the Tepper School of Business at Carnegie Mellon University, where the simulations were run. The research leading to these results has received funding from the Swiss National Science Foundation through the National Centre of Competence in Research (NCCR) in Robotics. 
Table 13: Maximum force per tendon of the human finger model.

\begin{tabular}{|c|c|}
\hline Tendon & Maximum force (N) \\
\hline flexor digitorum profundus (FDP) & 143.5 \\
\hline flexor digitorum superficialis (FDS) & 127.8 \\
\hline first dorsal interosseous (DI) & 145.6 \\
\hline extensor digitorum communis (EDC) & 48.7 \\
\hline extensor indicis proprius (EP) & 39.2 \\
\hline first palmar interosseous (PI) & 56.0 \\
\hline first lumbrical (LUM) & 12.6 \\
\hline
\end{tabular}

Table 14: Moment arms (mm) for each tendon of the human finger model about each degree of freedom.

\begin{tabular}{|c|c|c|c|c|}
\hline Tendon & MCP Abd & MCP flex & PIP flex & DIP flex \\
\hline FDP & -1.1 & 11.1 & 7.9 & 4.1 \\
\hline FDP & -1.7 & 11.9 & 6.2 & - \\
\hline DI & 6.1 & 3.7 & - & - \\
\hline EDC & 0.2 & -8.6 & -2.6 & -1.9 \\
\hline EP & -1.3 & -9.0 & -2.6 & -1.9 \\
\hline PI & -5.8 & 6.6 & -2.6 & -1.9 \\
\hline LUM & 4.8 & 9.3 & -2.6 & -1.9 \\
\hline
\end{tabular}

\section{Appendix}

\subsection{Human fingers moment arms}

Moment arms, and maximum force per tendon are given in tables $(1,2),[1,17]$.

More recent values of the human hand moment arms can be found in [6]. However, the authors do not mention in their paper the maximum force per tendon of their model.

\subsection{Joint limit and axes orientation constraints}

In order to have a grasp that is feasible for the hand, the corresponding joint angles $\left\{\theta_{i}^{j}\right\}, i=$ $1, . ., q^{j}, j=1, . ., 3$ should be within the joint limits. Two different representations of the joint angles were employed. The first one uses the non-linear functions cosine and sine in the computation of the kinematical chain. The second one replaces cosine and sine with the inner product between the relevant vectors. Let $c_{i}^{j}=\cos \left(\theta_{i}^{j}\right)$, and $s_{i}^{j}=\sin \left(\theta_{i}^{j}\right)$, we have the following relationships: 


$$
\begin{array}{ll}
c_{1}^{j}=\mathbf{v}^{j^{T}} \cdot \mathbf{r}_{2}^{j}, & \mathbf{s}_{1}^{j} \cdot \mathbf{r}_{1}^{j}=\mathbf{v}^{j} \times \mathbf{r}_{2}^{j} \\
c_{2}^{j}=\mathbf{r}_{1}^{j^{T}} \cdot \mathbf{e}_{2}^{j}, & \mathbf{s}_{2}^{j} \cdot \mathbf{r}_{2}^{j}=\mathbf{r}_{1}^{j} \times \mathbf{e}_{2}^{j} \\
c_{3}^{j}=\mathbf{e}_{2}^{j{ }^{T}} \cdot \mathbf{e}_{3}^{j}, & \mathbf{s}_{3}^{j} \cdot \mathbf{r}_{3}^{j}=\mathbf{e}_{2}^{j} \times \mathbf{e}_{3}^{j} \\
c_{4}^{j}=\mathbf{e}_{3}^{j} \cdot \mathbf{e}_{4}^{j}, & \mathbf{s}_{4}^{j} \cdot \mathbf{r}_{4}^{j}=\mathbf{e}_{3}^{j} \times \mathbf{e}_{4}^{j}
\end{array}
$$

Satisfying joint angle limits induce a limitation on $c_{i}^{j}$ and $s_{i}^{j}$. Their corresponding lower and upper bounds are respectively $c_{\text {low }}, s_{\text {low }}$ and $c_{u p}, s_{u p}$ :

$$
\begin{aligned}
& c_{\text {low }}^{j} \leq c_{i}^{j} \leq c_{\text {up }}^{j}, \quad i=1, . ., q^{j}, \quad j=1, . ., 3 \\
& s_{\text {low }}^{j} \leq s_{i}^{j} \leq s_{\text {up }}^{j}, \quad i=1, . ., q^{j}, \quad j=1, . ., 3
\end{aligned}
$$

For each finger $j$, the revolute joints responsible for flexion/extension have parallel axes and the abduction/adduction axis, defined by the vector $\mathbf{r}_{1}^{j}$ is orthogonal to $\mathbf{r}_{2}^{j}$. These constraints can be expressed by the following equations:

$$
\begin{gathered}
\mathbf{r}_{2}^{j}=\mathbf{r}_{3}^{j}=\mathbf{r}_{4}^{j}, \quad j=1, \ldots, 3 \\
\mathbf{r}_{1}^{j^{T}} \cdot \mathbf{r}_{2}^{j}=0, \quad j=1, \ldots, 3
\end{gathered}
$$

\subsection{Friction cone segments}

The friction cone is linearized by a polyhedral convex cone with $m$ sides $\mathbf{l}_{i}^{j}$. In the reference frame of the object, $\mathbf{l}_{i}^{j}$ is given by:

$$
\mathbf{l}_{i}^{j}=R_{h} \cdot R_{q^{j}}^{j} \cdot\left[\begin{array}{c}
\mu \cdot \cos \left(\frac{2 \pi i}{m}\right) \\
1 \\
\mu \cdot \sin \left(\frac{2 \pi i}{m}\right)
\end{array}\right], \quad \text { where } i=1, . ., m
$$

Where $\mu$ is the friction coefficient. Vectors $\mathbf{l}_{i}^{j}$ are then normalized as follows: $\mathbf{l}_{i}^{j}=\frac{\mathbf{l}_{i}^{j}}{\left\|\mathbf{l}_{i}^{j}\right\|}$. That assumes that all the finger forces have the same limit.

\section{References}

[1] K. N. An, Y. Ueba, E.Y. Chao, W.P. Cooney, and R.L. Linscheid, Tendon excursion and moment arm of index finger muscles, Joumal of Biomechanics, vol. 16, pp.419-425, 1983. 
[2] V. Arsigny, P.Fillard, X. Pennec, and N. Ayache, Log-Euclidean metrics for fast and simple calculus on diffusion tensors, Magnetic Resonance in Medecine, vol. 56, no. 2, pp. 411-421, 2006.

[3] C. Melchiorri, Multiple whole-limb manipulation: An analysis in the force domain, Robotics and Autonomous Systems, vol. 20, pp. 15-38, 1997.

[4] C. Borst, M. Fischer and G. Hirzinger,Grasp planning: How to choose a suitable task wrench space, IEEE International Conference on Robotics and Automation, Vol. 1, pp. 319-325, 2004.

[5] J. Aleotti and S. Caselli, Interactive teaching of task-oriented robot grasps, Robotics and Autonomous Systems 58: pp. 539-550, 2010.

[6] O.I. Franko, T.M. Winters, T.F. Tirrell, E.R. Hentzen, and R.L. Lieber,Moment arms of the human digital flexors, Joumal of Biomechanics, vol. 44, pp.1987-1990, 2011.

[7] W. B. Griffin, R. P. Findley, M. L. Turner and M. R. Cutkosky, Calibration and mapping of a human hand for dexterous telemanipulation, Symposium on Haptic Interfaces for Virtual Environments and Teleoperator Systems, 2000.

[8] H. Hu, X. Gao, J. Li, J. Wang, and H. Liu, Calibrating human hand for teleoperating the HIT/DLR hand, Proceeding of IEEE International Conference on Robotics and Automation (ICRA), vol.5, pp. 4571- 4576, 2004.

[9] P.R. Kraus, V.I. Kumar, and P. Dupont., Analysis of frictional contact models for dynamic symulation., IEEE International Conference on Robotics and Automation, 1997.

[10] P.G. Kry and D.K. Pai, Interaction Capture and Synthesis, in Proceedings of ACM SIGGRAPH, vol. 25:(3), pp. 872-880, 2006.

[11] S. El-Khoury, M. Li and A. Billard, On the Generation of a Variety of Grasps, Robotics and Autonomous Systems, vol. 61(12), pp. 13351349, 2013.

[12] Z. Li and S.S. Sastry,Task-oriented optimal grasping by multifingered robot hands, IEEE Journal of Robotics and Automation, Vol. 4:(1), 1988.

[13] M. Malvezzi, G. Gioioso, G. Salvietti, D. Prattichizzo, and A. Bicchi, SynGrasp: A MATLAB toolbox for grasp analysis of human and robotic hands, IEEE International Conference on Robotics and Automation, 2013.

[14] B. Mirtich and J. Canny, Easily computable optimum grasps in 2D and 3D, IEEE International Conference on Robotics and Automation, vol. 1, pp. 739-747, 1994

[15] D.J. Montana, The condition for contact grasp stability, IEEE International Conference on Robotics and Automation, pp. 412-417, 1991.

[16] N.S. Pollard, Parallel algorithms for synthesis of whole-hand grasps, IEEE International Conference on Robotics and Automation, vol. 1, pp. 373-378, 1997.

[17] N. S. Pollard and R. C. Gilbert, Tendon Arrangement and Muscle Force Requirements for Humanlike Force Capabilities in a Robotic Finger, IEEE International Conference on Robotics and Automation, ICRA 2002.

[18] N. S. Pollard and A. Wolf, Grasp synthesis from example: tuning the example to a task or object, Workshop on Multi-point Interaction in Robotics and Virtual Reality, IEEE International Conference on Robotics and Automation, pp. 77-90, 2004.

[19] C. Rosales, L. Ros, J.M. Porta and R. Suarez, Synthesizing grasp configurations with specified contact regions, International Journal of Robotics Research, vol. 30, 2011.

[20] B. Siciliano and O. Khatib, Springer Handbook of Robotics, part D, chapter 28, 2008.

[21] R. Suárez, M. Roa, and J. Cornella, Grasp quality measures, Universitat Politècnica de Catalunya, Institut d'Organització i Control de Sistemes Industrials, Technical Report IOC-DT-P, 2006.

[22] A. Wächter and L.T. Biegler, On the Implementation of an Interior-Point Filter Line-Search Algorithm for LargeScale Nonlinear Programming, Mathematical Programming, vol. 106, no. 1, pp. 25-57, 2006. 